\title{
Electromagnetic Flow around Two Non-conducting Particles and the Interaction Forces —Different Diameter Cases-
}

\author{
Takuma OGASAWARA, Noboru YOSHIKAWA, ${ }^{11}$ Shoji TANIGUCHI" and Toshifumi ASAI ${ }^{21}$ \\ Formerly Graduate Student at Tohoku University, now at NEC-Tokin Inc., Asashi, Shiroishi, Miyagi 989-0223 Japan. \\ 1) Graduate School of Environmental Studies, Tohoku University, aza-Aoba, Aramaki, Aoba-ku, Sendai, Miyagi 980-8579 Japan. \\ 2) Elf Inc., Miyahara, Yodogawa-ku, Osaka 532-0003 Japan.
}

(Received on November 27, 2002; accepted in final form on February 22, 2003)

\begin{abstract}
In this study, electromagnetic (em) migration forces exerted to two non-conducting particles having different diameters are investigated, which are in conducting liquid with imposition of DC em field. The electromagnetically-driven flow (em-flow) around the particles was analyzed numerically. Its influence on the interaction force between the particles and on the magnitude of em migration forces (Gc) were examined for the two particles in three configurations; Direction of two particles in a row is either parallel to the current (Case 1), or to the magnetic field (Case 2), or to the em force (Case 3).

Four circulating flows were formed around the two particles. Strong penetrating flow between the particles existed in Case 1 and 2, but they had different flow directions. In Case 3, the penetration flow was weak. The migration force exerted to the small particle was influenced markedly. In Case 1, Gc of small particle is larger than that of the large particle twice as much. In Case 2, the direction of em migration force was opposite to the large particle. These relative differences in em migration force correspond to the repulsive interaction between the particles. Interaction forces along directions of the particles in the row were negligibly small in all three cases.

Nature of generating interaction forces between the particles was discussed by considering the pressure distribution on the particle surface.
\end{abstract}

KEY WORDS: electromagnetic force; flow; particle; interaction; migration force; two particles; different diameter.

\section{Introduction}

In order to produce clean steel, removal of nonmetallic inclusion from molten steel has been demanded. ${ }^{1)}$ Application of em force is one of the positive methods ${ }^{2-5}$ to accomplish this. Their principle is based on the theory proposed by Leenov and Kolin (L-K). ${ }^{6}$ They considered a single spherical particle or a cylinder having infinite length, placed in conducting liquid with imposition of DC em field. In this field, direction of the magnetic field is perpendicular to that of the electric current, thus the direction of em force is perpendicular to both directions. They calculated the em migration force by analyzing the em-flow around the particle. This analysis has been further modified by some researchers. Patel et al. ${ }^{7)}$ analyzed migration force to a single particle in magnetic field having various angles with respect to the current direction. Using numerical technique, $\mathrm{Da} \mathrm{Shu}^{8)}$ recently analyzed the single particle with wall proximity effect, and they also studied the problem of the two particles with their direction in a row parallel to em force (same diameters, Case 3).

There are a little studies done for the plural particles, although they are more likely in the actual non-metallic inclusions in liquid steel. Simulation model to describe the behavior of the plural particles is needed to be developed.
We started with the analysis for the two particle model, as its first stage. In our previous study, ${ }^{9)}$ interaction force and the migration forces between two non-conducting particles in three different configurations were calculated without considering the em-flow. In the following study, ${ }^{10)}$ their forces were calculated with consideration of em-flows around two particles. As a result, magnitude of the migration forces (Gc: Geometric coefficient, migration force divided by the bulk em force and by the volume) varied with the configuration of the two particles with respect to the DC em field, however, the interaction force such as attractive or repulsive force between the two particles were negligibly small in all the cases examined.

The previous studies employed two particles having the same diameter. In these cases, flow and em pressure distribution are symmetric around the two particles. This state was pointed out for the reasons of the no interaction force between the particles. In this study, two particles having different diameters are placed in three different configurations, then the em-flow and the em migration forces are calculated. The results are compared with our previous studies on the same diameter models. 


\section{Calculation Method}

Detailed calculation methods are described in the other report. ${ }^{10)}$ Thus, a brief description is given in this section.

\subsection{Fundamental Equations and the Boundary Con- ditions}

Two particles with different diameters are placed in a cell, as schematically shown in Fig. 1. Direction of the current, the magnetic field and the em force correspond to $+x,+z$ and $-y$, respectively. In this study, a diameter of one particle was taken larger than the other particle twice as much. They were placed with the row directions parallel to the three directions.

Because the particles are non-conducting, no body forces are generated in the particles. Instead, em force is generated in the surrounding conducting liquid, which causes the migration force having opposite direction to the bulk em force. Em-flow is induced because of the local current disturbance around the particles. This flow is the other contributing factor to the migration force. In this study, it is intended that the em flow around the particles are analyzed and the total migration force to the particles are calculated.

Electric current distributions around the particles are obtained by solving the Laplace equation (Eq. (1)) and Ohm's law (Eq. (2)) with the boundary conditions (Eqs. (3) and (4)), where $\phi$ is the electric potential and $\sigma$ is the electric conductivity. On the particle surface, boundary condition is expressed as Eq. (4), where $\mathbf{n}$ is the normal direction to the spherical surface of the particles.

$$
\begin{gathered}
\nabla^{2} \phi=0 \ldots \\
\mathbf{j}=-\sigma \nabla \phi . . \\
\mathbf{j}_{\mathrm{n}}=0 \ldots . \\
\frac{\partial \phi}{\partial \mathbf{n}}=0 . \\
\mathbf{f}_{\mathrm{em}}=\mathbf{j} \times \mathbf{B} .
\end{gathered}
$$

In order to set the bulk electric current, constant electric potential values were given to the upper and the lower surfaces of the cell and a constant potential gradient was given to the side wall. For the obtained current distributions, homogeneous magnetic field of $\mathbf{B}$ is imposed to the analyzed region and the em force is calculated according to Eq. (5).

Flow is induced locally around the particles because of the em force. The velocity distributions are obtained from the below Navier-Stokes equations of Eqs. (6) and (7):

$$
\nabla \cdot \mathbf{v}=0 \text {. }
$$

$$
\rho \frac{d \mathbf{v}}{d t}=\rho \frac{\partial \mathbf{v}}{\partial t}+\rho \mathbf{v} \cdot \nabla \mathbf{v}=-\nabla p+\eta \nabla^{2} \mathbf{v}+\mathbf{f}_{\mathrm{em}}
$$

Solving the flow equation, distributions of $p$ (pressure) and $v$ (velocity) were obtained. $F_{\mathrm{em}, \mathrm{p}}$ the migration force to the particles are consisted of three terms, the electromagnetic body force $\left(\mathbf{F}_{\mathrm{v}}\right)$, the electromagnetic pressure plus the inertia force due to the flow $\left(\mathbf{F}_{\mathrm{s}, \text { pre }}\right)$ and the viscous stress force $\left(\mathbf{F}_{\mathrm{s}, \mathrm{vis}}\right)$ as shown in Eq. (8), however, the volume force of the first term does not exist in the present case, because of the non-conducting particles.

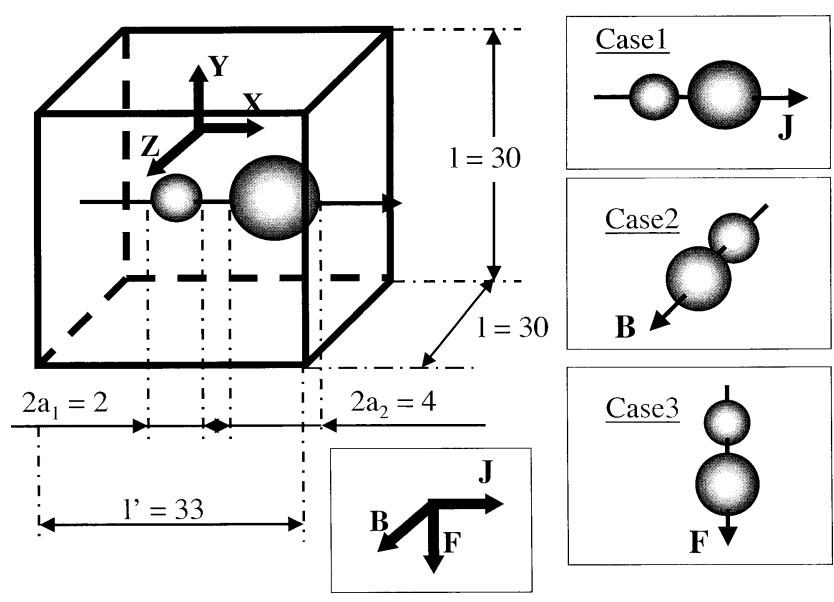

Fig. 1. Schematic illustration of cell dimension and configuration of particles for numerical calculation. Two particles with different diameters are placed in the cell. Stacking direction of the particles are parallel to current (case 1), to magnetic field (case 2) and to electromagnetic force (case 3). Length unit is meter.

$$
\mathbf{F}^{\mathrm{em}}=\mathbf{F}_{\mathrm{v}}^{\mathrm{em}}+\mathbf{F}_{\mathrm{s}, \mathrm{pre}}^{\mathrm{em}}+\mathbf{F}_{\mathrm{s}, \mathrm{vis}}^{\mathrm{em}}
$$

The second term is calculated by integrating the normal (diagonal) components of the electromagnetic pressure tensor and the inertia tensor due to convection over the particle surface, as given by Eq. (9). And the third term is calculated by integrating the viscous stress tensor over the particle surface, as given by Eq. (10). The viscous stress tensor is given by Eq. (11).

$$
\begin{array}{r}
\mathbf{F}_{\mathrm{s}, \mathrm{pre}}=\iint \mathbf{p} d \mathbf{S}=\iint p \cdot \mathbf{n} d S \\
\mathbf{F}_{\mathrm{s}, \text { vis }}=\iint \mathbf{T} \cdot d \mathbf{S} \ldots \ldots \ldots . . \\
\mathbf{T}=\eta \cdot\left(\frac{\partial v_{i}}{\partial x_{j}}+\frac{\partial v_{j}}{\partial x_{i}}\right) \ldots \ldots . .
\end{array}
$$

\subsection{Calculation Procedure}

Distributions of electric current were calculated by using a software ELFIN, which is based on the mixed analysis of the charge simulation method ${ }^{11)}$ and the surface simulation method. ${ }^{12)}$ Flow was analyzed using a software Fluent (ver. 5.2). Regions for the flow analysis were divided into meshes, especially finer in the area between the particles and the vicinities of the particle surfaces. The number of meshes were of an order of several hundred thousands. Coordinates of mesh points for the flow analysis were input into ELFIN, then the electric field and the current were calculated for each point. Em force distributions were calculated by a cross-product between the homogeneous magnetic field B and the electric current at each mesh point. This force field distribution was input into the flow analysis as the source term.

In the calculation, fundamental equation (Eq. (7)) was converted into non-dimensional form of Eq. (12), using parameters expressed in Eq. (13).

$$
\overline{\mathbf{v}} \cdot \bar{\nabla} \overline{\mathbf{v}}=-\bar{\nabla} \bar{p}+\alpha \overline{\mathbf{f}}_{\mathrm{em}}+\bar{\nabla}^{2} \overline{\mathbf{v}}
$$




$$
\begin{gathered}
\bar{\nabla}=\nabla \cdot(2 a), \quad \overline{\mathbf{v}}=\frac{\rho \cdot(2 a)}{\eta} \cdot \mathbf{v}, \quad \bar{p}=\frac{\rho \cdot(2 a)^{2}}{\eta^{2}} \cdot p, \\
\overline{\mathbf{f}}_{\mathrm{em}}=\frac{\mathbf{f}_{\mathrm{em}}}{\mathbf{f}_{\infty}}, \quad \alpha=\frac{\rho \cdot(2 a)^{3}}{\eta^{2}} \cdot \mathbf{f}_{\infty} \ldots \ldots \ldots \ldots \ldots \ldots
\end{gathered}
$$

In the present analysis, it was required to use the simplest values as possible for the sake of better convergence in iterations of Fluent analysis. Although the parameter were input using a unit of meter, value of $\alpha$ was selected to enable consideration of the actual non-metallic inclusions in steel. The input values are listed in Table 1.

The surface integrals expressed by Eqs. (9) and (10) were evaluated using the calculating functions in Fluent.

\section{Results}

\subsection{Em-flow State around Two Particles in Com- parison with the Previous Models}

The calculated flow patterns are shown in Fig. 2. They are patterns of (a) a single particle, (b) two particles with same diameters and (c) two particles with different diameters. Two particles are in Case 1 configuration. There are four circulating flows around the single particle (Fig. 2(a)), which are predicted in the analysis by L-K, and are a characteristic feature of the present DC em field problems. The confronted pairs of the circulating flows have the same directions, however, the other pairs have different flow directions. This feature was also observed in the case of the emflow around two particles with the same and different diameters (Figs. 2(b) and 2(c)). In the pattern of the same diameters (Fig. 2(b)), the upward flows (opposite direction of the em-flow) exist in both sides of the particles, however, the downward flow (same direction as the em force) pair extends over the scale of the two particles. In the pattern of the different diameters (Fig. 2(c)), the downward flow was

Table 1. Specific values used for calculation.

\begin{tabular}{|l|c|}
\hline Density, $\rho / \mathrm{kg} \cdot \mathrm{m}^{-3}$ & 1 \\
\hline Viscosity, $\eta / \mathrm{Pa} \cdot \mathrm{s}$ & 1 \\
\hline Electromagnetic force, $\mathrm{f}_{\mathrm{em}} / \mathrm{N} \cdot \mathrm{m}^{-3}$ & 1 \\
\hline Particle Diameter, $\mathrm{a} / \mathrm{m}$ & 1 \\
\hline \multicolumn{1}{|c|}{$\alpha$} & $8\left(\mathrm{a}_{1}=1\right), 32\left(\mathrm{a}_{2}=2\right)$ \\
\hline
\end{tabular}

not extended but localized to the large particle. The upward flows still exist in both sides of two particles.

The another characteristic feature in two particle cases is the existence of the penetrating flow between the particles. According to the previous results on the same diameter, ${ }^{10)}$ the flow direction is upward in the Case 1 , however, the direction is opposite in Case 2. The penetrating flow was weaker in Case 3 configuration, because of the geometric reason. The same tendency was observed in the flow patterns of the different diameter cases.

Velocity vector distributions of the different diameter cases are shown in Fig. 3 for the three configurations. Difference in directions of the penetrating flows is obvious (Figs. 3(a) and 3(b)) and the penetrating flow is weak (Fig. 3(c)). The flow velocities are different in both sides of the particles. The velocity distribution in Case 1 is plotted along the row direction in Fig. 4, comparing with the same diameter case.

Comparing with the same diameter case (having the same diameter with the small particle), about three times larger velocity magnitude is present in the right side of the large particle, and twice larger velocity in the left side of the small particle. According to the L-K analysis, the larger velocity in em-flow is generated in a particle having the larger diameter. Although diameter of the small particle is not different from the same diameter case, larger velocity was generated around the small particle. This is because of the influence of the large particle. The penetrating flow velocity is also enhanced and becomes larger than the same diameter case, as can be seen in Figs. 3 and 4.

\subsection{Magnitude of Em-migration Force (Gc)}

The Gc values were calculated by integration of the pressure on the particle surface. The obtained values are listed in Table 2. The interaction force between the particles such as the attractive or repulsive force $\left(F_{\mathrm{p} x}^{\mathrm{em}}\right.$ components in $x$-direction (Case 1), $F_{\mathrm{p} z}^{\mathrm{em}}$ components in $z$-direction (Case 2) and $F_{\mathrm{p} y}^{\mathrm{em}}$ components in $y$-direction (Case 3$)$ ) was shown to be negligibly small, comparing with that of $F_{\mathrm{p} y}^{\mathrm{em}}(y$-direction, the principle migration force component (interaction direction in Case 3)). Although there is lager em-flow than the same diameter cases and the flow states were not symmetric, the interaction force was not generated in the different diameter models, either. Larger em migration force (to
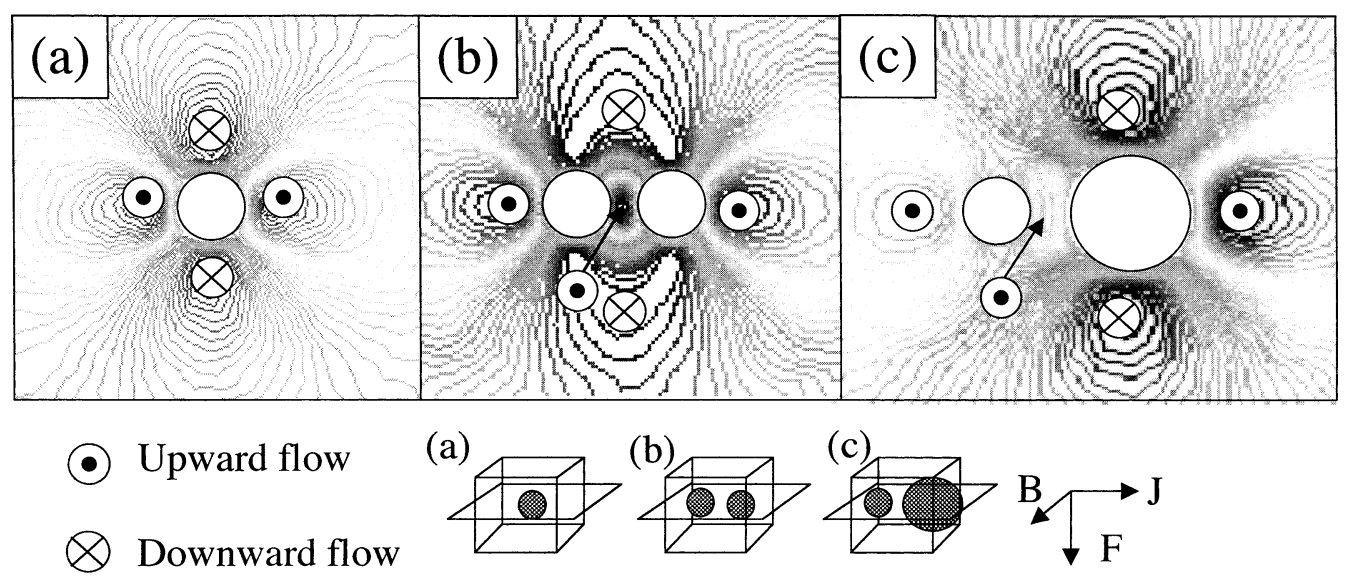

Fig. 2. Flow patterns (a) around a single particle, (b) around two particles with the same diameter and (c) around two particles with different diameter. Two particles in (b) and (c) are in Case 1 configuration. 


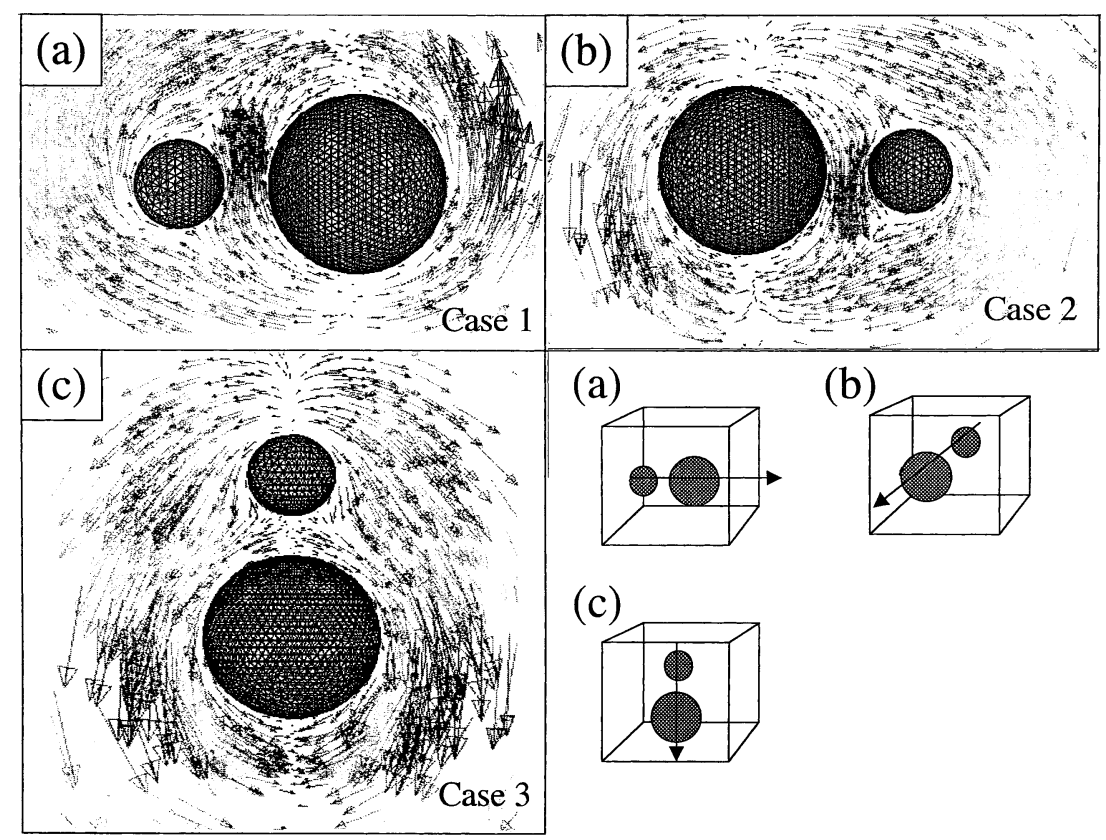

Fig. 3. Velocity vector distributions around two particles with different diameter in three configurations.

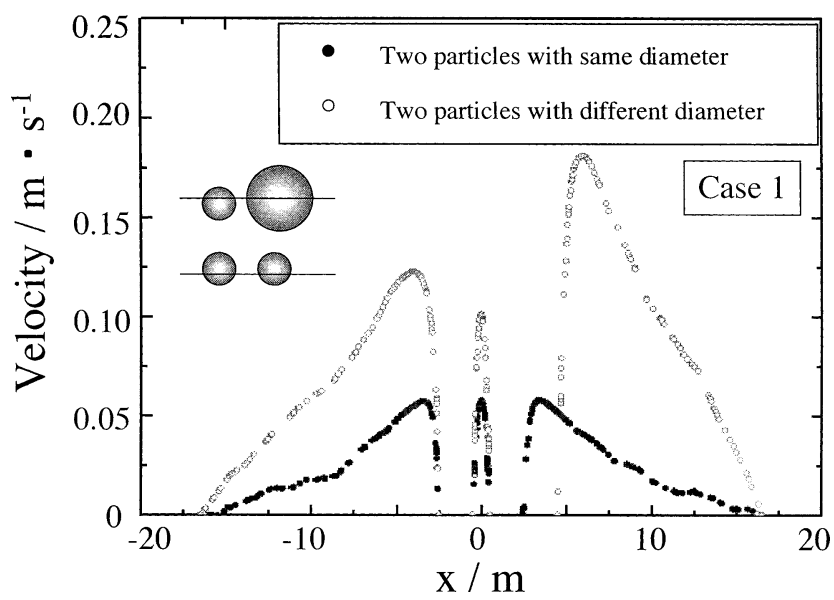

Fig. 4. Velocity distributions around two particles having same diameter and different diameter along the cross sections shown in the schematic drawing in the figure.

Table 2. Gc values obtained by calculation. Interaction force components are shown in bold letters.

\begin{tabular}{|c|c|c|c|}
\hline & case 1 & case 2 & case 3 \\
\hline x-component (large) & $\mathbf{0 . 0 0 4 4 5}$ & 0.02118 & 0.0023 \\
\hline y-component (large) & 0.74811 & 0.763 & $\mathbf{0 . 7 6 7 4}$ \\
\hline z-component (large) & -0.0015 & $\mathbf{0 . 0 0 4 4 8}$ & 0.00041 \\
\hline x-component (small) & $\mathbf{- 0 . 0 0 4 3}$ & -0.0056 & 0.00134 \\
\hline y-component (small) & 1.55441 & -0.0559 & $\mathbf{0 . 8 3 5 1 7}$ \\
\hline z-component (small) & 0.00329 & $\mathbf{- 0 . 0 0 1 9}$ & -0.0076 \\
\hline
\end{tabular}

the em force direction $\left.\left(F_{\mathrm{p} y}^{\mathrm{em}}\right)\right)$ is exerted to the large particle than to the small one. However, because the Gc values are calculated by division of the force by the volume, the small particle has the larger Gc value than the large particle, eventually.

The relationship between Gc values (Gc: $y$ component) and the inter-particle distance obtained for three cases (the same diameter cases) are plotted in the left graph of Fig. 5. In this figure, our first results of the two particles without considering em-flow ${ }^{10)}$ are plotted with open marks and the results by considering the em-flow ${ }^{9)}$ are plotted with closed marks. Dependence of Case 2 has different tendency from the other two cases. The em-flow effect on the Gc was positive in Case 1 (to increase Gc), negative in Case 2, and much small in Case 3. This tendency was caused by the difference in the penetrating flow direction in each case.

In Fig. 5(a) (same diameter case), data of one particle are plotted because Gc values were almost same between the two particles. However, in the different diameter case, Gc values are different in each particle. They are all plotted in the right graph (b). The large particle had similar Gc values in all cases, namely between 0.75 and 0.85 . On the other hand, Gc value of a small particle became very large in Case 1, and became negative in Case 2. The Case 2 result indicates the obvious repulsive state along the em force $(y$-) direction and the Case 1 configuration also has the repulsive state, relatively. In Case 3 , Gc value was not different from that of the large particle very much.

The obtained results on the Gc relationships for the different configurations are summerized in Fig. 6. In Case 3, when the small particle is above the large particle, the interaction was relatively repulsive and it was relatively attractive when the small particle is beneath the large particle, although the interaction forces are very weak for both cases.

\section{Discussion}

\subsection{Influence of Em-flow States on the Gc Magnitude}

Relationship between inter-particle distance and Gc values was obtained in our previous study ${ }^{9)}$ without considering em-flow. In this analysis, the result of the Case 2 was shown different from the other cases. The reason has been discussed in analogy of the cylinder cases analyzed by L-K. Namely, distortion of the electric current to the em force direction is generated only in Case 2 cylinder, and this distortion is important for cancellation of the migration force.

In the succeeding study, ${ }^{10)}$ it was reported that the em- 


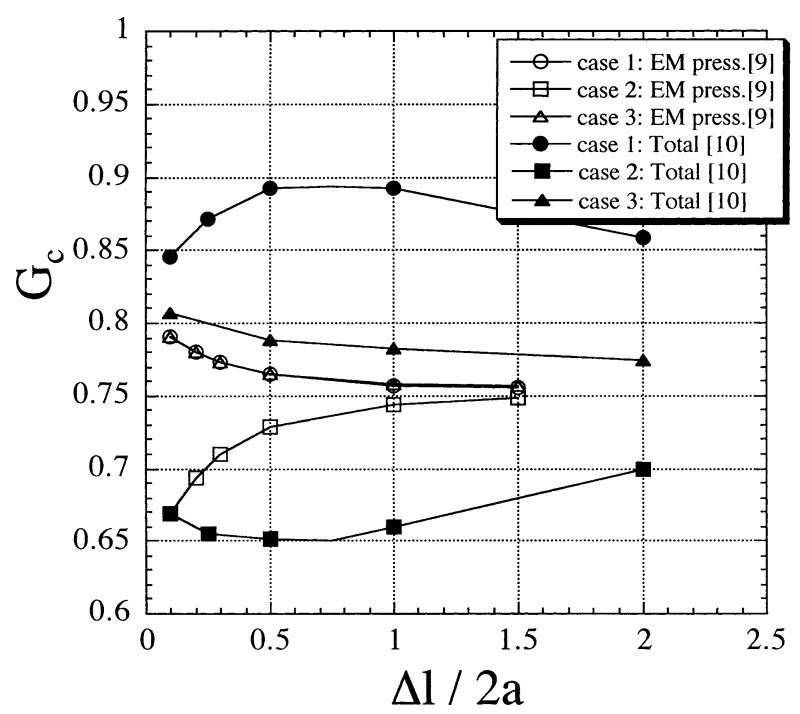

(a)

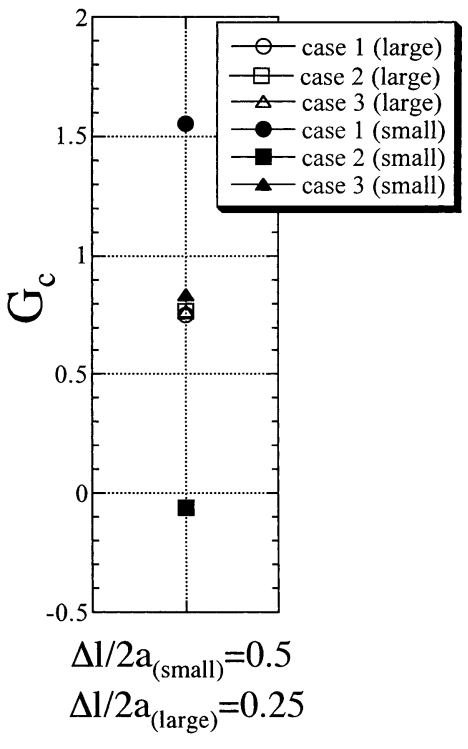

(b)

Fig. 5. (a) Relationship between particle distance and Gc (magnitude of em-migration force, $y$ component) (same diameter cases). (b) Gc values of two particles with different diameters.

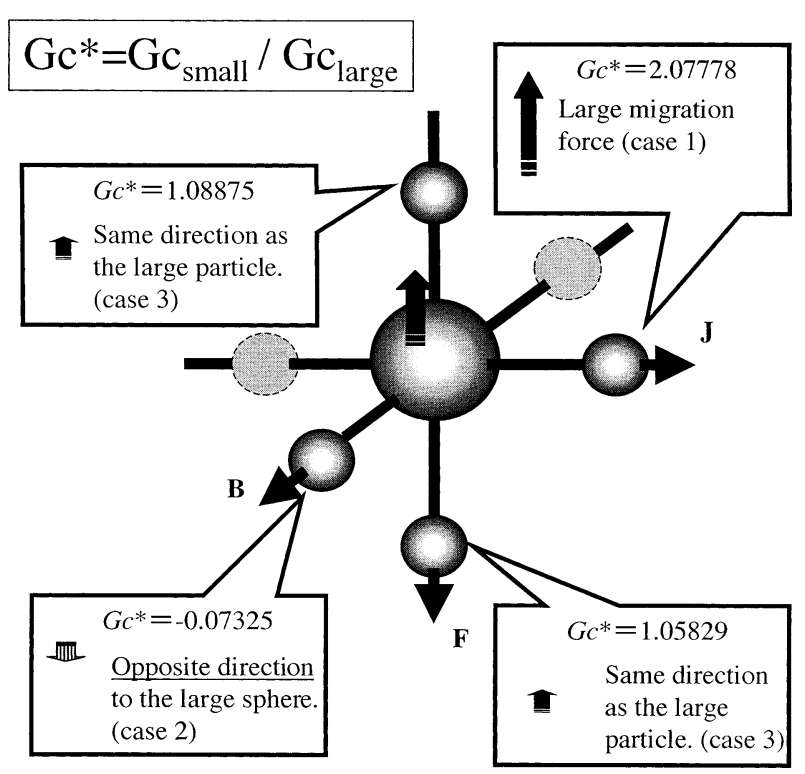

Fig. 6. Relationship between the particle configuration and the Gc ( $y$ component) values.

flow around the particles influenced the em migration forces (Gc values). Although the contribution of the viscous stress is about $10 \%$ of the em pressure, their dependence was significant in Case 1 and 2, however, the influence was weak in Case 3. The Gc values were dependent on the penetrating flows, as mentioned in Sec. 3.2.

The em-flow around a single particle becomes large as an increase of the particle size.

$$
v=\left(\frac{k}{2 r^{3}}\right)\left(\frac{a^{2}}{r^{2}}-1\right)\left(x y \mathbf{e}_{x}+\left(z^{2}-x^{2}\right) \mathbf{e}_{y}-y z \mathbf{e}_{z}\right) \ldots
$$

This is predicted in the analysis by L-K, as shown in Eq. (16).

The velocity magnitude at certain position $(x, y, z)$ has a dependence on the radius $(a)$ to about square power. In the present case, however, the enhancement of the velocity around large particle was less than four times (twice larger diameter) (Fig. 4). This might be due to existence of the other particle.

In this study (different diameter cases), a pair of the circulating flow was formed in both sides of the particles, and the small particle was wrapped in the flow field caused by the large particle. Therefore, effect of the flow on Gc value was larger in the small particle than expected from its diameter. Magnitude of the penetrating flow also increased because of the large particle. The flow states influenced the small particle to increase Gc twice larger than the large particle in Case 1, and moreover caused opposite direction of the em migration force with respect to the larger particle in Case 2, because of the difference in the penetrating flow direction.

\subsection{On the Nature of the Interaction Force}

It was demonstrated that the interaction forces between two particles along their row direction was negligibly small in all the cases, less than about one percent of the em migration force along the em force direction ( $y$-direction). In this section, reasons for the obtained results are discussed. Interaction forces in Case 1 configuration is obtained by integrating $F_{\mathrm{p}, x}$ ( $x$-component of surface force vector, $F_{\mathrm{p} x}=$ $(p \cdot \mathbf{n})_{x}, p$ : pressure (scalar), $\mathbf{n}$ : surface normal vector) over the particle surface by Eq. (10). In order to obtain the insight for formation of the migration force, distributions of $F_{\mathrm{p}, x}$ component on the particle surface are compared.

First, a single particle in a homogeneous pressure field, $F_{\mathrm{p}, x}$ distribution on the particle surface has a function form of $\sin \phi$ where a variable $\phi$ is an angle from the $y$-axis to the normal direction of the reference position on the surface (Fig. 7(a)). The distribution is symmetric with respect to $y$ axis, namely, two sine curves in the positive and negative regions are the same. Thus, integration along the two integral paths gives equivalent values but having opposite signs. They cancel out, indicating no interaction force to $x$ - 

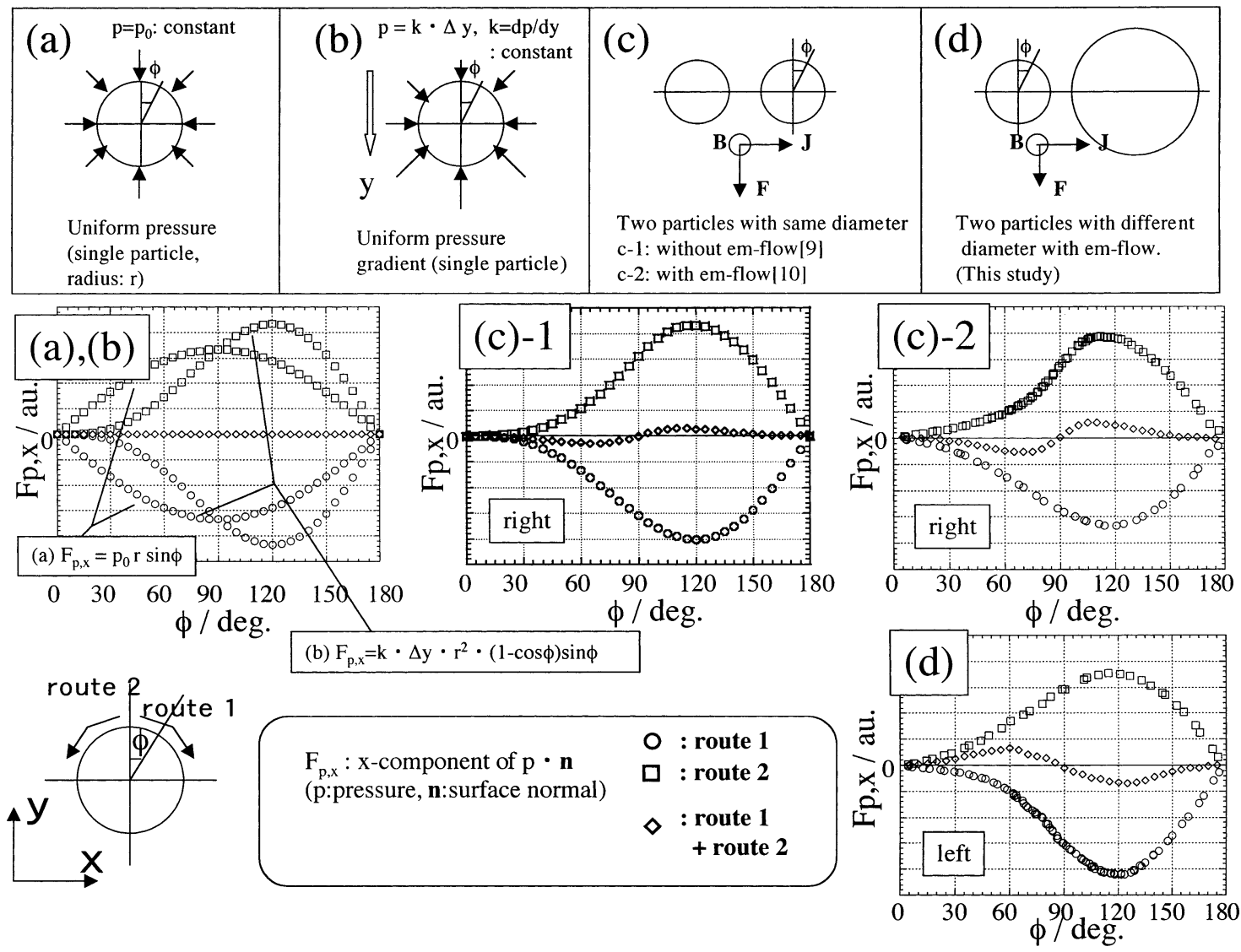

Fig. 7. Pressure force distributions around particles in various cases.

direction.

Second, a single particle in a pressure field having a constant pressure gradient (Fig. 7(b)), $F_{\mathrm{p}, x}$ has a function form of $k \Delta y r(1-\cos \phi) \sin \phi(k$ is a constant having a dimension of pressure divided by length.). This case corresponds to a single particle with imposition of DC em pressure field (without em-flow). In this case, the peak of the curve exists at $\phi=120 \mathrm{deg}$. Because the curves are also symmetric, their summation becomes zero and no force is generated in $x$-direction.

Third, two particles in DC em field, in which em-force is exerted to $-y$ direction (opposite to em migration force). $F_{\mathrm{p}, x}$ distribution without considering the em-flow is shown in Fig. 7(c)-1. In this case, peaks of both curves exist almost at $\phi=120$ deg., however, their curves have a little difference in shape, due to the modified em pressure field by existence of the other particle. The summation of both curves is plotted with a diamond mark. This curve has nonzero value, however, the total summation is almost canceled to become zero, eventually. Thus, the interaction force is also negligible.

In the same model with considering em-flow (Fig. 7(c)2), $F_{\mathrm{p}, x}$ distribution curve in the clearance side (positive part, route 2) has larger distortion, and the peak is also deviated from $120 \mathrm{deg}$. It can be seen that the summation curve (diamond mark) has larger values than (c)-1 case. In spite of the larger distortion in $F_{\mathrm{p}, x}$ curve due to the viscous stress of the em-flow, the summation curves also cancel each other and the interaction force is also negligible.

Lastly, the present case (different diameter) is shown in Fig. 7(d). A plot for the small particle is shown, because the particle has the same diameter as the case (c). In this case, the clearance is in route 1 side, corresponding to the negative curve. The curve has distortion, however, the peak position is almost at $120 \mathrm{deg}$. The deviation of the summation plots is rather smaller than (c)-2 case, in spite of the larger modification of the flow field. Therefore, the interaction force naturally resulted in almost zero.

Here, a question is raised why no interaction force is generated between two particles ( $x$-direction, in Case 1 configuration), namely why the $F_{\mathrm{p}, x}$ distributions cancel in the two integral routes. The reason might be attributed to the followings: Generation of interaction force needs the loss of symmetry in $F_{\mathrm{p}, x}$ (pressure field) distribution between route 1 and 2, which originally has to be brought about either by the distortion of em force or by distribution of the flow field. However, it has to be noted that the pressure field is determined by integration of the em force field (and the flow stress field). Moreover, the em migration force to $x$-direction is calculated by integration of the $F_{\mathrm{p}, x}$ (x-component of the pressure). Twice integration is needed. Therefore, considerably large change in em force is required to raise the pressure field alteration, and then to $x$-component (interaction) migration force. According to these arguments, the flow field alteration due to the different diameter particles was not enough to cause the alteration of the pressure field and the migration forces.

As the last comment, on testifying the validity of the present analysis, measurement of the fluid velocity distribution around particles by means of Laser Doppler method is taken into consideration, using aqueous solution. And it will be possible to measure the degree of em migration 
forces by balancing with the buoyancy force, as performed in the previous study. ${ }^{9)}$ However, it will be not necessarily easy to resolve the localized flow velocity around the particles and it is difficult to measure the migration forces, separately.

The analyzed results in Fig. 6 suggest that the migration forces exerting to the particles (with different diameters) in the neighborhood differ from each other. Thus, they are expected to rearrange into the energetically stable configurations, which might be one of the factors related to coagulation of the particles. This is a favorable phenomenon for removing non-metallic inclusions from liquid steel, because of the increased size of the (coagulated) particle causing larger migration force.

\section{Conclusion}

(1) Similar to the same diameter cases, four circulating flows existed in the em-flow around two particles with different diameters. The upward flow existed in the sides of both particles, however, the downward flow localized to the large particle. Penetrating flow existed between the particles, which was upward in Case 1, downward in Case 2 and weak in Case 3.

(2) Interaction forces between large and small particles were also negligible in comparison with the em migration force to $+y$ direction. However, the em migration force to $y$ (em force)-direction varied with the particle configurations. In Case 1, the small particle has larger Gc value of twice as much. And in Case 2, Gc has the opposite sign to that of larger particle. These features indicate the repulsive force acting to $y$-direction between the particles.

(3) Reason for the large migration force to small particle is due to the fact that the large em-flow caused by the large particle wraps the small particle. Non considerable interaction force between the particles was discussed consid- ering the pressure field distribution on the particle surfaces.

\section{Acknowledgements}

This work was financially supported by Research Fund for Iron and Steel Making, ISIJ. and Grant-in-Aid for Scientific Research on Priority Areas (B), Japanese Ministry of Education, Culture, Sports, Science and Technology. The authors are grateful for technical advice by Dr. M. Nyu and Mr. Y. Tokuyama at Fluent Asia Pacific, Ltd. Precious discussion by Prof. Ueno at Tohoku Univ. is acknowledged. Cooperative works by students (Mr. Y. Kubota, K. Hongo and Mr. J. Nakano) are also acknowledged.

\section{REFERENCES}

1) T. Gladman: Ironmaking Steelmaking, 19 (1992), No. 6, 457.

2) S. Taniguchi and J. K. Brimacombe: ISIJ Int., 34 (1994), No. 9, 722.

3) J. Park, J. Morihara, K. Sassa and S. Asai: Tetsu-to-Hagané, 80 (1994), 389

4) Y. Tanaka, K. Sassa, K. Iwai and S. Asai: Tetsu-to-Hagané, 81 (1995), 1120.

5) A. Alemany, J. P. Argous, J. Barbet, M. Ivanes, R. Moreau and S. Poinsot: French Patent No. 804004430, (1980).

6) D. Leenov and A. Kolin: J. Chem. Phys., 22 (1954), No. 4, 683.

7) A. D. Patel and N. El-Kaddah: Proc. Int. Symp. on Electromagnetic Process. Mater., (EPM1994), ISIJ, Tokyo, (1994), 115.

8) Da Shu, B. D. Sun, J. Wang, T. X. Li, Z. M. Xu and Y. H. Zhou: Metall. Mater. Trans. B, 31B (2000), 1527.

9) Y. Kubota, N. Yoshikawa and S. Taniguchi: Tetsu-to-Hagané, 87 (2001), 1.

10) T. Ogasawara, N. Yoshikawa, S. Taniguchi and T. Asai: "Flow of conducting liquid around two non-conducting particles in DC electromagnetic field and the electromagnetic migration force", submitted to Mater. Metall. Trans.

11) The Institute of Electrostatics Japan, Handbook of Electrostatics (in Japanese), Ohbun-sha, Tokyo, (1981) 181. or K. Hidaka: Charge Simulation Method: User's guide (Version 8), University of Tokyo, Japan, (1994)

12) T. Kono and T. Takuma: Numerical Electric Field Calculations (in Japanese), Corona, Tokyo, (1980). 